\title{
Spectral Dependence of CMB Polarization and Parity
}

\author{
K.R.S. Balaji* \\ Department of Physics, McGill University, Montréal, Québec, H3A 2T8, Canada \\ Robert H. Brandenberger ${ }^{\dagger}$ \\ Department of Physics, Brown University, Providence, RI 02912, USA \\ Damien A. Easson ${ }^{\ddagger}$ \\ Department of Physics, Syracuse University, Syracuse, NY 13244-1130, USA
}

(Dated: December 5, 2018)

\begin{abstract}
The polarization of the cosmic microwave background radiation (CMBR) can serve as a probe for nonstandard parity violating interactions. Many such interactions are predicted in particle physics models arising from theories with extra dimensions such as superstring theory. These interactions produce an optical activity that depends on the space-time nature of the parity violating field. In particular, it is possible to obtain a frequency-dependent differential rotation of the polarization axis. The form of the frequency dependence is sensitive to the time evolution of the parity odd background field. Thus, one may be able to study a broad class of parity violating operators through polarization measurements of the CMBR.
\end{abstract}

PACS numbers: 98.80.Cq.

\section{INTRODUCTION}

Perhaps the single most important source of observational data in cosmology is the cosmic microwave background radiation (CMBR). The observed radiation was last scattered around a red-shift of $z=1100$ (the time of "recombination") and carries a tremendous amount of information about the physics of the early universe. It is expected that through such cosmological data extensions of new physics (normally insensitive to the electroweak scale) such as string theory or any other alternative theories may establish contact with observation.

The spectral distribution of the CMBR is to very high accuracy [1] that of a black body. Primordial density fluctuations seed anisotropies in the CMBR, and the physics at the surface of recombination generates a polarization of these anisotropies (for a review see e.g. [2]). In this Letter, we point out that many parity-odd terms in the effective Lagrangian for low energy physics lead to a frequency-dependent rotation of the polarization axis. In fact, the details of the frequency dependence of this rotation may give insight into the nature of the parity-odd terms, and therefore provide an avenue to probe the high energy fundamental physics which is responsible for the parity-odd terms in the low energy effective action.

Our analysis builds on early work by Carroll, Field and Jackiw who pointed out that parity-odd terms in the effective Lagrangian, e.g. a Chern-Simons term [3] or a term coupling a new scalar field $\phi$ (such as the scalar fields used to model quintessence) to $F \wedge{ }^{*} F$ [4] leads

\footnotetext{
*Email:balaji@hep.physics.mcgill.ca

${ }^{\dagger}$ Email:rhb@het.brown.edu

‡Email:easson@physics.syr.edu
}

to the rotation of the polarization axis of the radiation from distant quasars. Carroll then studied the bounds on quintessence models coming from the observational bounds on this effect [5]. Lue, Wang and Kamionkowski [6] (and independently Lepora [7]) pointed out that the coupling terms mentioned above will also produce a rotation in the polarization axis of the CMBR ("cosmological birefringence") and that this effect can be used to probe parity-violating interactions. The new aspects of our work are the focus on the frequency dependence of the effect, and the application of this result to the study of fundamental physics. The frequency dependence of the birefringence discussed here is different from that produced by Faraday rotation, and hence can be tested experimentally.

In order for new terms $\mathcal{L}_{i}$ in the effective Lagrangian to produce cosmological birefringence, the terms in the fundamental theory leading to $\mathcal{L}_{i}$ must possess electromagnetic (EM) interactions. To be specific, let us consider parity-odd Chern-Simons (CS) terms of a given form. A couple of examples are (i) a three-form Kalb-Ramond field (see e.g. [8]) which is ubiquitous in string models and (ii) a one-form pseudo-scalar field [3]; both of these can have electromagnetic interactions.

It is interesting to study the nature of the parity violating interactions by looking at the qualitative and quantitative features of the cosmological birefringence. For instance, what multiplies ${ }^{*} F$ in the new term $\mathcal{L}_{i}$ of the effective Lagrangian can be a form $F_{j}$ of various dimensionalities, not just a one form as assumed in previous work. Can one determine the nature of this form $F_{j}$ from the specifics of the frequency dependence? In this Letter, we neglect the space-dependence of $F_{j}$ (which is well justified to leading order in our context of cosmology). We show that the predicted birefringence has a spectral dependence with signatures that depend on the form $F_{j}$. In 
particular, the time dependence of the underlying fields plays a crucial role.

We conclude that the polarization of the CMBR can be sensitive to any derivative couplings in the parity-odd operator in the effective Lagrangain. Since a frequency dependence of the rotation of the polarization axis is predicted in many cases and since it carries information about the underlying fundamental physics, we propose that it is of interest to perform a spectral analysis of any birefringently polarized CMBR.

\section{A GENERAL ANALYSIS}

Our starting point consists of adding to the Maxwell equations a term coming from the interaction of the EM gauge field $A_{\mu}$ with an arbitrary parity odd CS field $\mathcal{O}_{\mu}$. The interaction Lagrangian is given by

$$
\mathcal{L}=\mathcal{O}_{\mu} A_{\nu} \tilde{F}^{\mu \nu}
$$

where $\tilde{F}^{\mu \nu}$ is the dual of the usual antisymmetric EM field tensor. The resulting field equations are [3]

$$
\nabla_{\mu} F^{\mu \nu}=(\mathcal{O} \cdot \tilde{F})_{\nu} ; \nabla_{\mu} \tilde{F}^{\mu \nu}=0 .
$$

Due to gauge invariance, $\mathcal{O}$ must be curl free and hence expressible in terms of a gradient of a scalar. In general, it is either of the type

$$
\mathcal{O}_{\mu} \sim H_{\mu \nu} \partial_{\nu} \phi
$$

or a term with more derivatives

$$
\begin{aligned}
& \mathcal{O}_{\mu} \sim H_{\mu \lambda \sigma \ldots n}\left[\partial_{\lambda} \phi \wedge \partial_{\sigma} \phi \ldots \wedge \partial_{n} \phi\right] \text { or } \\
& \mathcal{O}_{\mu} \sim H_{\mu \lambda \sigma \ldots n}\left[\partial_{\lambda} \phi \partial_{\sigma} \phi \ldots \partial_{n} \phi\right] .
\end{aligned}
$$

Here, $H$ denotes a tensor field (in general independent of the fields which we have already introduced) which is either symmetric or antisymmetric and can have spacetime dependence, $H \equiv H(\eta, x)$. In the simplest case of (2.3), $\mathcal{O}_{\mu}$ is the covariant derivative of a scalar field like the axion [10].

The physical process we focus on in this Letter which arises due to the above new parity-odd interaction term is cosmological birefringence, the rotation of the plane of polarization of the CMBR. Another effect (to be discussed elsewhere) is a temperature and polarization anisotropy due to a fluctuating $H$ field.

Let us analyze the consequence of $(2.2)$ when $\mathcal{O}$ is a spatially homogeneous operator with $H \equiv H(\eta)$. We choose a spatially flat Friedmann-Robertson-Walker background metric $d s^{2}=a^{2}(\eta)\left(-d \eta^{2}+d x_{i} d x^{i}\right)$ where $a(\eta)$ is the scale factor of the Universe and $\eta$ is conformal time. In this case (2.2) translates to

$$
\begin{aligned}
\frac{\partial}{\partial \eta}\left[a^{2}(\eta) \vec{E}\right]-\nabla \times\left[a^{2}(\eta) \vec{B}\right] & =-2 C\left(\frac{d \Phi}{d \eta}\right)^{n}\left[a^{2}(\eta) \vec{B}\right] \\
\frac{d \Phi}{d \eta} & =H^{1 / n} \frac{d \phi}{d \eta} \equiv \Phi^{\prime}
\end{aligned}
$$

Here, $n$ denotes the number of derivatives in $\mathcal{O}$, and the constant $C$ (used to specify the dependence in (2.3) and (2.4)) is determined by the mass scale $M$ characteristic of the physics leading to the effective Lagrangian (2.1), i.e. $C \sim M^{1-d}$ where $d$ is the dimension of the fields of the operator $\mathcal{O}$ appearing in (2.4). Setting $F_{ \pm}=a^{2}(\eta) \vec{B}_{ \pm}(\eta)$, leads to the differential wave equation

$$
\frac{d^{2} F_{ \pm}}{d \eta^{2}}+\left(k^{2} \mp 2 k C \Phi^{\prime n}\right) F_{ \pm}=0 .
$$

If $\Phi^{\prime} \neq 0$, the left- and right-circular modes ( $\mp$ states) of the photon are split, leading to a modified dispersion relation

$$
\omega_{ \pm}^{2}=k\left[k \mp 2 C\left(\Phi^{\prime}\right)^{n}\right] .
$$

Therefore, if the photons travel for a time interval $\delta \eta$, they obtain a differential rotation (birefringence) given by

$$
\delta \theta=C\left(\phi^{\prime}\right)^{n} H(\eta) \delta \eta+C \delta H(\eta)\left(\phi^{\prime}\right)^{n} \delta \eta .
$$

The first term in (2.8) arises due to the presence of the $\phi$ field while the second term arises due to the variation in $H(\eta)$ over the time slice $\delta \eta$. The latter term also gives rise to a change in the photon energy as can be seen from (2.7). Therefore, the differential rotation $(d(\delta \theta) \equiv d \theta)$ is correlated to the change in the photon energy. This effect is purely due to the time dependence of $H(\eta)$. Thus, the resulting optical birefringence can become a function of the frequency change, an effect which to our knowledge has not been pointed out (see e.g. [6]). We note that this effect can also occur if the $\phi$ field is a temporally oscillating massive field while $H(\eta)$ is constant. This possibility can be trivially examined along the lines of the present analysis. As described in (2.8), it is important to observe that the rotation leading to a change of the photon energy is an additional contribution to an existing rotation due to the $\phi$ field. Note also, that according to (2.8), the rotation angle $\theta$ depends on the amplitudes of both the $\phi$ and $H$ fields. Since it depends linearly on the constant $C$, it follows that the birefringence due to higher dimensional operators is suppressed by the corresponding powers of $M$. Assuming that the field $\phi$ is massless, we have (neglecting the expansion of the Universe)

$$
\phi(\eta)=\dot{\phi}_{0} \cdot \eta+\phi_{0},
$$

where $\dot{\phi}_{0}$ and $\phi_{0}$ are constants. For this case, substituting for $C$, the rotation angle (2.8) becomes

$$
\theta(\eta)=\left[H(\eta)\left(\phi^{\prime}\right)^{n} M^{1-d}\right] \eta .
$$

\section{SPECTRAL AND TIME DEPENDENCE}

In this section, we study the frequency dependence of the cosmological birefrigence identified in the previous section. 


\section{A. Effects for $\eta_{<} \eta_{r e c}$}

First, let us elaborate on the electromagnetic effects that occur for all interaction times $\eta$ before the time of recombination $\eta_{\text {rec }}$. These are not actually observable today. In the next subsection we discuss the conversion to effects observable at times $\eta \geq \eta_{\text {rec }}$.

Let us begin by clarifying what we mean by spectral dependence of the differential birefringence in (2.8). Physically, due to the presence of the time-dependent background field, the photon undergoes two simultaneous effects:

- (i) an optical birefringence due to the $H(\eta)$ field (along with the $\phi$ field), and

- (ii) a variation of the photon energy due to its interaction with the time varying $H(\eta)$ field.

The second feature is seen by Taylor expanding (2.7)

$$
\frac{d \omega_{ \pm}}{d \eta} \approx \mp C\left(\frac{d \phi}{d \eta}\right)^{n} \cdot \frac{d H(\eta)}{d \eta} \neq 0 .
$$

In the above, we have assumed that the field $\phi$ is massless, and thus (2.9) holds. If this were not the case, and the time dependence of $\phi$ were nonlinear, then there would be an extra term in (3.11) proportional to $H$ and to the second derivative of $\phi$. We stress that the above change in energy is due to interactions at all times $\eta<\eta_{\text {rec }}$. Clearly, if the background field were constant, then a change in the rotation would not cost the photon any energy. In other words, the scattering leading to the optical birefringence would be an elastic process. Then, the rotations described here would be frequency independent. For an inealstic process, the change in the photon energy $\left(d \omega_{ \pm}\right)$associated with a differential rotation $(d \theta)$ is

$$
\frac{d \omega_{ \pm}}{d \theta}=\frac{d \omega_{ \pm}}{d \eta} \cdot \frac{d \eta}{d \theta} \text { for all } \eta \leq \eta_{r e c},
$$

where all derivatives are taken at constant value of the wavenumber $k$. Using (2.8) and (3.12) we find

$$
\frac{d \omega_{ \pm}}{d \theta}=\mp \frac{H^{\prime}}{H^{\prime} \eta+H} ; H^{\prime}=\frac{d H(\eta)}{d \eta} .
$$

The above result allows us to infer the time dependence of $H(\eta)$ by inverting (3.13). We obtain

$$
\frac{d H(\eta)}{H(\eta)}=\frac{f_{ \pm} d \eta}{1 \mp f_{ \pm} \eta} ; f_{ \pm} \equiv \frac{d \omega_{ \pm}}{d \theta} .
$$

Integrating (3.14) leads to

$$
\log \left(\frac{H(\eta)}{M_{X}}\right)=\int \frac{f_{ \pm} d \eta}{1 \pm \eta f_{ \pm}}: \eta<\eta_{r e c} .
$$

Here, $M_{X}$ can be identified with the scale at which the electromagnetic interaction is defined.

\section{B. Observable effects for $\eta_{\geq} \eta_{r e c}$}

What is actually observable today is a spectral/frequency dependence in the rotation angle. In principle, this dependence is a correlation of an observable energy width to a given change in the rotation angle. The actual observable change in rotation is

$$
d \tilde{\theta}=\frac{d \theta}{d \omega_{ \pm}} \cdot d \omega_{ \pm}=f_{ \pm}^{-1} d \omega_{ \pm} \text {for all } \eta>\eta_{r e c} .
$$

Note that the effect vanishes if the scattering process is elastic, i.e. if $d \omega_{ \pm}=0$. The tilde in (3.16) denotes the actual observable rotation. Therefore, for a measurable frequency width $\delta \tilde{\omega}$, the true spectral dependent differential rotation is

$$
\frac{d \tilde{\theta}}{d \tilde{\omega}}=f_{ \pm}^{-1} \cdot \frac{d \omega_{ \pm}}{d \tilde{\omega}}
$$

As a consistency check, (3.17) indicates that if no spectral dependence of the differential rotation $\left(d \omega_{ \pm} \rightarrow 0\right)$ were observed, we would deduce from (3.15) that $H(\eta)$ is a constant field $\sim M_{X}$. In order to obtain a frequency dependent birefringence one requires a background field which is time dependent. Finally, using (2.7) and (3.17)

$$
\frac{d \tilde{\theta}}{d \tilde{\omega}}=\frac{C\left(\phi^{\prime}\right)^{n}}{d \tilde{\omega}} \cdot \int_{\eta}^{\eta_{r e c}} f_{ \pm}^{-1} \cdot \frac{d H(\eta)}{d \eta} d \eta,
$$

where the integration runs over a finite short time interval from $\eta$ to $\eta_{\text {rec }}$ during which the photons are scattering.

We illustrate the above results for a specific choice of $H(\eta)$, namely $H(\eta) \sim h \cdot \eta$. This could arise (neglecting the expansion of the Universe) if $H$ were a massless field obeying the equation $\nabla^{2} H(\eta)=0$, and taking $H$ to be spatially homogeneous. In the linearized approximation, using (3.13), we find that, for an infinitesimal energy interval $\Delta \omega$, the left and right modes rotate by an amount

$$
\Delta \theta_{ \pm}=\mp 2 \eta \Delta \omega_{ \pm} \Rightarrow f_{ \pm}^{-1}=\mp 2 \eta \text {. }
$$

This indicates that the differential rotation (for $\eta<\eta_{\text {rec }}$ ) grows with energy and is independent of the density of the background field. For this scenario, we find from (3.18) the observable differential rotation to be

$$
\frac{d \tilde{\theta}}{d \tilde{\omega}}=\mp \frac{C h\left(\phi^{\prime}\right)^{n}}{2 d \tilde{\omega}} \eta_{r e c}^{2} .
$$

Note that the observable spectral dependence vanishes for $\eta=\eta_{\text {rec }}$ since in this case there are no scattering events that lead to a birefringence. Naively, it also appears from (3.20) that in order to obtain a large and thus observable effect, the time width in scattering $\left(\eta-\eta_{\text {rec }}\right)$ needs to be large. However, in this case our neglect of the expansion of the Universe is no longer justified (this could easily be corrected by taking into account the Hubble friction in the equation of motion for $H$ and using the 
resulting time dependence). More importantly, the incoherence of the scattering events would need to be taken into account, and this would alter the amplitude of the predicted signal. However, on the positive side, we could easily retain a signal from physics in the very early Universe, at times $\left(\eta \ll \eta_{\text {rec }}\right)$, have the field $H(\eta)$ depend on time in the very early Universe but be frozen in the late Universe. In this case, there would be no cosmological constraints on the scenario coming from demanding that the kinetic energy of the $H$ field be negligible in the late Universe.

Let us conclude this section by evaluating the order of the magnitude of the differential birefringence in the case $n=1$. Taking $\phi$ to be dimensionless, and $H$ to have the canonical dimension 1 , we obtain

$$
\frac{d \tilde{\theta}}{d \tilde{\omega}} \sim \frac{M_{2}}{M} \frac{\eta_{r e c}^{2}}{\eta_{1} \eta_{2}} \frac{1}{d \tilde{\omega}},
$$

where $M_{2}$ is the amplitude of $H$ and $\eta_{1}$ and $\eta_{2}$ are the time scales of the change in $\phi$ and $H$, respectively. Here, we have set the dimensionless amplitude of $\phi$ to 1 . For massless fields, we expect the time scales are given by the Hubble time, and thus the ratio of times in (3.21) is of the order one. Hence, logarithmic differential birefringence can be of the order 1 if $M_{2} \sim M$ when $\phi$ is measured in units where $\phi=1$ at $\eta=\eta_{r e c}$. In turn, this will be the case if the new physics only contains one new mass scale.

One may be concerned that the polarization signature could be washed out by Compton scattering while the photons are in thermal equilibrium [19]. However, note the potential depolarization effects due to Compton scattering only compete with the cosmic birefringence at early times when the photons are in thermal equilibrium. The depolarization effects rapidly decrease at later times. Now, there is a large time interval between when the photons fall out of equilibrium and when they last scatter, and it is during this time interval (which corresponds to the finite thickness of the last scattering surface) that our polarization effect can build up.

Demanding that the kinetic energy in $\phi$ and $H$ not destroy the successful predictions of nucleosynthesis will lead to bounds on the ratio of masses (typically, $M_{2}$ will have to be small compared to $M$ in order that the condition is satisfied). However, the details of the bounds will depend on the model in several ways, e.g. on the form of the kinetic energy term for the new fields, the history of the time dependence of the field, etc.. We do not see any obvious reason why our effect should be so small as to be unmeasurable in upcoming polarization experiments.

\section{A STRING BASED MODEL}

The goal of this section is to demonstrate that interaction terms of the type postulated at the beginning of this paper arise in theories of fundamental physics. To be specific, consider the relevant parts of a ten dimensional type-IIB superstring bosonic action (see e.g. [11] for a recent review). In addition to the terms in the action coming from the graviton, the dilaton and the NS-NS [20] two form (these terms give the action for dilaton gravity with the addition of a term involving the square of the field strength of the NS-NS two form), there are terms of the form

$$
\begin{aligned}
S_{\text {IIB }} & =-\frac{1}{2 \kappa_{0}^{2}} \int d^{10} x \sqrt{-g}\left\{\frac{1}{12}\left(G^{(3)}+C^{(0)} B^{(3)}\right)^{2}\right\} \\
& +\frac{1}{8 \kappa_{0}^{2}} \int d^{10} x B^{(2)} C^{(2)} G^{(3)} B^{(3)},
\end{aligned}
$$

where we have adopted the usual notation. Thus, $G^{(3)}=$ $d C^{(2)}$ is the field strength of a R-R two form $C^{(2)}, C^{(0)}$ is a zero-form R-R scalar field while $B^{(3)}$ is the field strength of the NS-NS two form. In what follows we will assume that the dilaton is fixed.

Consider the first term in (4.22). The four-dimensional reduced action giving the kinetic term for the $B$ field is

$$
S=\frac{1}{12} \int d^{4} x \sqrt{-g}\left(C^{(0)}\right)^{2} B_{\mu \nu \alpha} B^{\mu \nu \alpha},
$$

where $B_{\mu \nu \alpha}=\partial_{[\mu} B_{\nu \alpha]}$, and $B_{\mu \nu}$ is the Kalb-Ramond field. In the context of cosmology and, in particular, in the case of the Pre-Big-Bang scenario (for a recent review see [13]) the role of such a term has been studied, see e.g. $[12]$. Fixing the scalar field $C^{(0)}$, the equation of motion becomes

$$
\partial_{\nu}\left(\sqrt{-g} B^{\mu \nu \alpha}\right)=0
$$

and is satisfied by introducing the dual axion field $\sigma$,

$$
B^{\mu \nu \alpha}=\frac{1}{\sqrt{-g} C} \epsilon^{\mu \nu \alpha \beta} \partial_{\beta} \sigma .
$$

Then, the action (4.23) becomes

$$
S=\frac{1}{2} \int d^{4} x \sqrt{-g} g^{\alpha \beta} \partial_{\alpha} \sigma \partial_{\beta} \sigma .
$$

The electromagnetic interaction is obtained through the interference term $\left(G^{(3)} B^{(3)}\right)$, which gives the low energy four dimensional action

$$
S=\frac{1}{2} \int d^{4} x \sqrt{-g} g^{\alpha \beta} B^{\mu \nu \alpha} A_{\mu} \tilde{F}_{\nu \alpha} .
$$

It can be easily shown that for (4.27) the operator $\mathcal{O}_{\mu}$ corresponds to a space-time constant $H$ field with $\mathcal{O}_{\mu}=\partial_{\mu} \sigma$. Such an interaction leads to a frequency independent birefringence.

Next, let us consider the second term in (4.22) which yields the four dimensional effective action

$$
S=\frac{3}{4} \int d^{4} x B \partial_{\nu} \sigma\left(A_{2 \lambda} \tilde{F}_{1}^{\nu \lambda}-A_{1 \lambda} \tilde{F}_{2}^{\nu \lambda}\right) .
$$

Identifying the linear combination $\left(A_{1 \lambda}+A_{2 \lambda}=\sqrt{2} A_{\lambda}\right)$ as the photon field, we obtain the effective action for the electromagnetic interaction

$$
S=\frac{3}{2} \int d^{4} x B \partial_{\nu} \sigma A_{\lambda} \tilde{F}^{\nu \lambda} .
$$


Clearly, (4.29) reduces to our original action (2.1) with the identification $\mathcal{O}_{\mu}=H \partial_{\nu} \sigma$. In this case $H \equiv B$ and $\sigma$ are independent massless scalar fields, and the $B$ field is taken to be the zero-form scalar field which arises after compactifying the higher dimensional two-form field $B^{(2)}$. We note that the model has two massless scalar fields, the axion and an additional field $H$. The cosmological significance of the string model outlined here essentially follows along the lines discussed for the axion field [14].

In summary, we have shown that terms in the Lagrangian such as the ones we have used in our analysis appear quite naturally in string theory models. This provides a concrete example of how string theory may ultimately make contact with experiment.

\section{V. $T-E$ CROSS CORRELATION AND SHAPE}

Spatial field fluctuations lead to density perturbations which in turn induce CMB temperature anisotropies. Here we show that the parity-odd interactions discussed in this paper lead to specific signals in the temperaturepolarization cross correlation function of the CMBR sky [21].

The cosmic sky can be mapped in terms of temperature $(T)$ and polarization $(Q)$ parameters. The polarization can be divided into a a curl-free piece (the $E$-modes) and a divergence-free piece (the $B$-modes). The first observation of the $E$-mode polarization and the $T-E$ cross correlation was made by the DASI experiment [16]. Recently, WMAP has produced data for the $T-E$ cross correlation at a much larger significance [15]. These signals result due to physics of last scattering (with $z \sim 1000$ ) and hence should be most prominent at smaller angular scales $\left(\leq 2^{\circ}\right)$.

Conventionally, the temperature and polarization maps are decomposed into spherical harmonics. The weight factors of this expansion lead to nonzero moments $C_{l}^{X X^{\prime}}$ (for a multipole $l$ ). Here, $X$ and $X^{\prime}$ can be $T, E$ or $B$, thus yielding auto-correlation $\left(X=X^{\prime}\right)$ and crosscorrelation $\left(X \neq X^{\prime}\right)$ functions. In the limit of strict parity invariance, one expects the cross-correlations involving $B$ to vanish. However, as in our case, if there is a parity violating background field, then due to the rotation of the photon plane of polarization by an amount $\theta$ there will result a cross-correlation

$$
C^{T B}=C^{T E} \sin \theta .
$$

Therefore, if there is frequency dependent rotation, we find,

$$
\frac{d C^{T B}}{d \tilde{\omega}_{ \pm}}=-C^{T E}\left(\frac{d \theta}{d \tilde{\omega}_{ \pm}}\right) \cos \theta
$$

Thus, we conclude that the shape of $C^{T B}$ will be the same as that of $C^{T E}$ only if the rotations are frequency independent. If otherwise, we find an interesting modification which is determined by the nature of the background field. For instance, as in the case of massless fields, using (3.20) we find this modulation is

$$
\frac{d C^{T B}}{d \tilde{\omega}_{ \pm}}= \pm C^{T E}\left(\frac{C h\left(\phi^{\prime}\right)^{n} \eta_{r e c}^{2}}{2 d \tilde{\omega}}\right) \cos \theta
$$

\section{SUMMARY}

In summary, we have outlined the possibility of identifying parity odd electromagnetic interactions by observations of the CMBR. The first effect is a cosmological birefringence (rotation of the polarization axis) which in general will depend on the frequency. The second effect is a cross-correlation function $C^{T B}$ between the CMB temperature and the B-mode of the polarization, a correlation function whose frequency dependence is determined by the cross-correlation function $C^{T E}$ involving the Emode of the polarization. The relation between the two spectra depends on the specifics of the parity odd interactions. We have seen that a measurement of the differential rotation of the photon field can give information about the time dependence of the parity odd fields which enter in the interaction terms. In the present analysis we demonstrated this for the case of massless time varying fields.

In following the inflationary paradigm of early Universe cosmology, we presumed that the parity odd fields $H$ appearing in our interaction terms are spatially homogeneous. Note that in a more general setting where the space-dependence of $H$ is nontrivial, the spectral dependence of the birefringence is sensitive to the spatial distribution of the field. The details remain to be worked out. In the limit of $H$ being constant in space and time, we recover from (2.8) the result that the rotation of the polarization axis doe not depend on the frequency $[3,6]$. The frequency-dependent birefringence discussed here can be distinguished from the effects of Faraday rotation. The latter effects decrease in magnitude as the frequency increases $\left(\theta_{\text {Faraday }}(k)=C \times k^{-2}, C\right.$ being a constant), whereas our cosmological rotation of the polarization axis increases for larger frequencies. We anticipate that future CMB polarization measurements will be tuned to measure the spectral dependence of any observable cosmological birefringence discussed here.

\section{Acknowledgments}

We are grateful to Guy D. Moore for discussions and a careful reading of a preliminary version of this draft. We thank Horace Stoica, Greg Tucker and Mark Trodden for several helpful discussions. RB thanks the McGill theory group for hospitality during visits to Montréal during which this work was initiated. RB is supported in part 
by the US Department of Energy under Contract DEFG02-91ER40688, TASK A. DE is supported in part by NSF-PHY-0094122 and funds provided by Syracuse Uni- versity. KB is supported by NSERC (Canada) and by Fonds de recherche sur la nature et les technologies of Québec.
[1] J. C. Mather et al., Astrophys. J. 420, 439 (1994);

H. Gush, M. Halpern and W. Wishnow, Phys. Rev. Lett. 65, 537 (1990).

[2] W. Hu and M. J. White, New Astron. 2, 323 (1997) [arXiv:astro-ph/9706147].

[3] S. M. Carroll, G. B. Field and R. Jackiw, Phys. Rev. D 41, 1231 (1990).

[4] S. M. Carroll and G. B. Field, Phys. Rev. D 43, 3789 (1991).

[5] S. M. Carroll, Phys. Rev. Lett. 81, 3067 (1998) [arXiv:astro-ph/9806099].

[6] A. Lue, L. M. Wang and M. Kamionkowski, Phys. Rev. Lett. 83, 1506 (1999) [arXiv:astro-ph/9812088].

[7] N. F. Lepora, arXiv:gr-qc/9812077.

[8] S.J. Gates, M. Grisaru, M. Rocek, W. Siegel, Superspace (W.A. Benjamin, New York 1983).

[9] In the presence of a nonzero mass, one uses the WKB approximation for estimating the birefringence as in [3].

[10] P. Sikivie, Phys. Lett. B 137, 353 (1984).

[11] For a review see, C. V. Johnson, arXiv:hep-th/0007170.

[12] N. Kaloper, Phys. Rev. D 44, 2380 (1991).

[13] M. Gasperini and G. Veneziano, Phys. Rept. 373, 1 (2003) [arXiv:hep-th/0207130].
[14] In this case, we expect isocurvature perturbations and we refer to Stephen D. Burns, hep-ph/9711303 for a review. In addition, there can be some restrictions for this case as discussed by Silvia Mollerach, Phys. Lett. B242, 158 (1990).

[15] A. Kogut et al., Astrophys. J. Suppl. 148, 161 (2003) [arXiv:astro-ph/0302213].

[16] J. Kovac, E. M. Leitch, P. C., J. E. Carlstrom, H. N. W. and W. L. Holzapfel, Nature 420, 772 (2002) [arXiv:astro-ph/0209478].

[17] R. G. Crittenden, D. Coulson and N. G. Turok, Phys. Rev. D 52, 5402 (1995) [arXiv:astro-ph/9411107].

[18] R. Crittenden, J. R. Bond, R. L. Davis, G. Efstathiou and P. J. Steinhardt, Phys. Rev. Lett. 71, 324 (1993) [arXiv:astro-ph/9303014].

[19] We thank Guy D. Moore for raising this concern.

[20] Here, NS stands for Neveu-Schwarz, and R-R will later be used to denote Ramond-Ramond.

[21] The effects are similar to those due to tensor field fluctuations [17]. Indeed, if we assume the $H$ field to be traceless, the process mimics closely perturbations due to a gravitational system [18]. 